\title{
Electrohydrodynamics of a droplet in a highly confined domain: A numerical study
}

Cite as: Phys. Fluids 32, 123305 (2020); https://doi.org/10.1063/5.0028818

Submitted: 09 September 2020 . Accepted: 17 November 2020 . Published Online: 04 December 2020

Roozbeh Saghatchi, (D) Amin Rahmat, and (D) Mehmet Yildiz

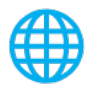

\section{Physics of Fluids GALLERY OF COVERS}




\title{
Electrohydrodynamics of a droplet in a highly confined domain: A numerical study
}

\author{
Cite as: Phys. Fluids 32, 123305 (2020); doi: 10.1063/5.0028818 \\ Submitted: 9 September 2020 - Accepted: 17 November 2020 • \\ Published Online: 4 December 2020
}

\author{
Roozbeh Saghatchi, ${ }^{1,2,3, a)}$ (D) Amin Rahmat, ${ }^{4, b)}$ (D) and Mehmet Yildiz ${ }^{1,2,3, c)}$ (D)
}

\begin{abstract}
AFFILIATIONS
${ }^{7}$ Faculty of Engineering and Natural Sciences, Sabanci University, Tuzla 34956, Istanbul, Turkey

${ }^{2}$ Integrated Manufacturing Technology Research and Application Center, Sabanci University, Tuzla 34956, Istanbul, Turkey

${ }^{3}$ Composite Technologies Center of Excellence, Sabanci University-Kordsa, Pendik 34906, Istanbul, Turkey

${ }^{4}$ School of Chemical Engineering, University of Birmingham, Birmingham B15 2T, United Kingdom
\end{abstract}

Note: This paper is part of the Special Topic on Turbulent and Multiphase Flows.

a) Electronic mail: roozbeh@sabanciuniv.edu

b) Electronic mail: a.rahmat@bham.ac.uk

${ }^{c)}$ Author to whom correspondence should be addressed: mehmet.yildiz@sabanciuniv.edu

\begin{abstract}
In this paper, electrohydrodynamics (EHD) deformation of a droplet in a highly confined domain is studied by using the incompressible smoothed particle hydrodynamics method. Simulations are performed for six different systems of a droplet and ambient fluid corresponding to different electrical properties. The effects of confinement ratios, from 0 to 0.95 , on the droplet deformation are discussed thoroughly. It is shown that the deformation is highly dependent on the ratios of electrical permittivity, electrical conductivity, and confinement ratio. To demonstrate the droplet behavior, electric force components on the droplet interface are calculated and discussed in detail. It is shown that the interaction of these forces plays a major role in the droplet deformation. Furthermore, it is illustrated that the pressure force becomes significant at high confinement ratios and affects the droplet behavior in addition to the electric forces. Different values of unbounded deformation are selected for the EHD simulation. The effect of unbounded deformation on the droplet behavior is also discussed, and it is found that the unbounded deformation influence is important in some of the systems and confinement ratios.
\end{abstract}

Published under license by AIP Publishing. https://doi.org/10.1063/5.0028818

\section{INTRODUCTION}

Electrohydrodynamics (EHD) of a suspended droplet is widely used in various engineering applications including droplet printing, ${ }^{1}$ electrocoalescence experiments in the petroleum industry, ${ }^{2} \mathrm{D}$ printing, ${ }^{3}$ biosensors, and bioengineering, ${ }^{4}$ among others.

Early theoretical studies were based on an extensive simplification where both fluid phases were treated either as perfect dielectrics (insulators) or perfect conductors. Such an assumption causes the droplet to deform only in the direction of the applied electric field (prolate deformation). ${ }^{5}$ However, Allan et al. experimentally observed in their pioneering study that the droplet could also elongate in the direction perpendicular to the electric field. ${ }^{6}$ Based on the observation of Allan and Mason, Taylor introduced the leaky dielectric model considering the fluids to be slightly conductive that allows for the accumulation of free electric charges on the interface. ${ }^{7}$ Employing this model to simulate the EHD related problems, several numerical studies have been conducted including Finite Volume Method (FVM), ${ }^{8}$ Finite Element Method (FEM), ${ }^{9}$ and Lattice Boltzmann Method (LBM). ${ }^{10,1}$

Although there have been many studies on the EHD of a droplet in an unbounded domain, ${ }^{12-16}$ the number of studies on confined domains is relatively low. The confined domain refers to the condition where the dimensions of both the droplet and the domain are comparable. The EHD deformation and manipulation of a confined droplet are widely observed in microfluidics and labon-a-chip devices, ${ }^{17}$ such as droplet microreactors, ${ }^{18}$ microfluidic mixing, ${ }^{19}$ electrocapillary, ${ }^{20}$ microfluidic droplet detection, ${ }^{21}$ and 
micro-segmented flow systems. ${ }^{22}$ For example, Esmaeeli and Behjatian analytically studied the steady-state deformation of a leaky dielectric droplet in confined 2D and 3D domains under an electric field. ${ }^{23,24}$ They investigated the effect of the confinement ratio on the droplet deformation and indicated that the confinement ratio leads to scaling the electric field and the free electric surface charge depending on the conductivity ratio. Nevertheless, these two analytical studies were based on the assumption of small droplet deformation, and bereft of investigating large deformations. Furthermore, the confinement ratios they studied were not as high as those typically observed in microfluidic systems. Santra et al. conducted a numerical study using commercial software (COMSOL Multiphysics) to simulate the EHD deformation of a confined single droplet and double droplets between two parallel electrodes. In their study, the confinement ratio was relatively high only in the direction of the applied electric field.

These studies claim that the magnitude of droplet deformation is affected by two main factors, namely, (a) the magnitude of the normal component of both electric and hydrodynamic stress and (b) the sign of the normal component of hydrodynamic stress, both of which are dependent on the confinement ratio. ${ }^{9,23}$ In these studies, however, no investigation of the electric force and its components has been reported. In general, the effect of the electric force on the droplet deformation is very complex and requires a detailed investigation of the force itself and its components as well to have a complete comprehension of the droplet behavior. Another major deficiency in those studies is the inaccurate definition of normal hydrodynamic stress. For example, in the study of Santra et al., velocity magnitude is employed to obtain the normal hydrodynamic stress ${ }^{9}$ disregarding the pressure effect. However, as will be shown in this study, pressure makes a significant contribution to the normal hydrodynamic stress and it has an indispensable influence on the droplet deformation, specifically at high confinement ratios. Although recent studies regarding the EHD of a confined droplet discuss novel physics, such as compound droplet dynamics ${ }^{25}$ and the transient behavior of droplets, ${ }^{26}$ the authors are not aware of any similar studies in the literature, which compare the competing electrohydrodynamic forces acting on the droplet interface in a confined system. We believe that analyzing the force fields acting on such complex systems will provide a deeper and more reliable understanding of the problem, which distinguishes our study from other similar studies.

Motivated by these considerations, in the present study, the deformation of a highly confined 2D leaky dielectric droplet under the applied electric field will be simulated using incompressible smoothed particle hydrodynamics (ISPH) methods. To see the impacts of electrical property ratios, six different systems of fluids will be employed. All the two main components of the electrical force will be calculated on the droplet interface, and their influence on the droplet deformation will be discussed thoroughly considering both the magnitude and sign of them. Furthermore, the pressure field will be calculated inside the domain, and its effect on the droplet deformation, particularly at high confinement ratios, will be discussed. In order to facilitate the analysis, a new parameter, namely, the force ratio $(F R)$, will be introduced and applied on the droplet interface to evaluate the influences of all related forces on the droplet deformation. Additionally, it will be shown that the analytical results are not reasonable at high confinement ratios, while our numerical method is able to calculate the physical and justifiable deformation values at high confinement ratios.

The organization of this paper is as follows. In Sec. II, the governing equation of the problem and its non-dimensional parameters are mentioned. In Sec. III, our numerical method, ISPH, is introduced and the governing equations are discretized into the ISPH framework. In Sec. IV, problem is defined and the accuracy of our numerical method is investigated by comparing it with the available data from other studies. In Sec. V, numerical results are presented for droplet behavior in the confined domain. Finally, concluding remarks are provided in Sec. VI.

\section{GOVERNING EQUATIONS}

Governing equations of an incompressible flow in the absence of gravity may be written as

$$
\begin{gathered}
\nabla \cdot \mathbf{u}=0, \\
\rho \frac{\mathrm{D} \mathbf{u}}{\mathrm{D} t}=-\nabla p+\frac{1}{\mathrm{Re}} \nabla \cdot \boldsymbol{\tau}+\frac{1}{\mathrm{We}} \mathbf{f}_{(s)}+\frac{1}{\mathrm{Ew}} \mathbf{f}_{(e)},
\end{gathered}
$$

where $\mathbf{u}, p, \rho$, and $t$ are the velocity vector, pressure, density, and time, respectively. $\nabla \cdot$ and $\nabla$ are the divergence and the gradient operators, respectively. The material time derivative is represented with $\mathrm{D} / \mathrm{D} t=\partial / \partial t+\mathbf{u} \cdot \nabla$. Here, $\boldsymbol{\tau}$ is the viscous stress tensor, which is defined as follows:

$$
\boldsymbol{\tau}=\mu\left[\nabla \mathbf{u}+(\nabla \mathbf{u})^{\dagger}\right]
$$

in which $\mu$ is the dynamic viscosity and superscript $\dagger$ is the transpose operation. Re, We, and Ew denote the Reynolds, Weber, and electroWeber numbers, respectively, which will be defined later.

To calculate the surface tension on the droplet interface, the continuum surface force (CSF) method is used,

$$
\mathbf{f}_{(s)}=\gamma \kappa \hat{\mathbf{n}} \delta
$$

where constant $\gamma$ is the surface tension coefficient, $\kappa=-\nabla \cdot \hat{\mathbf{n}}$ represents the interface curvature, where $\hat{\mathbf{n}}$ is the unit surface normal vector, and $\delta$ is the Dirac delta function.

The electrical force vector $\mathbf{f}_{(e)}$ is defined as ${ }^{28}$

$$
\mathbf{f}_{(e)}=-\frac{1}{2} \mathbf{E} \cdot \mathbf{E} \nabla \varepsilon+q^{v} \mathbf{E}
$$

in which $\mathbf{E}$ is the electric field vector, $\varepsilon$ is the electric permittivity, and $q^{v}$ denotes the volume charge density near the interface. It should be noted that this equation is written in the electrostatic units (CGS-ESU). The electrical force vector contains two distinct parts, the first and second terms on the RHS of (5), which are known as polarization and Coulomb force, respectively. Neglecting magnetic induction and assuming a small amount of dynamic current, the electric field is irrotational ${ }^{29}$ and can be calculated as

$$
\mathbf{E}=-\nabla \phi,
$$

where $\phi$ is the electric potential.

For the two-fluid systems with finite electric conductivities, one can assume that the electric relaxation time is fast compared to the viscous relaxation time, resulting in the following relations: 


$$
\nabla \cdot(\sigma \nabla \phi)=0
$$

$$
q^{v}=\nabla \cdot(\varepsilon \nabla \phi)
$$

in which $\sigma$ denotes the electric conductivity. The interface jump condition for Eq. (7) is the continuity of the current, ${ }^{14}$

$$
\|\sigma \mathbf{E}\| \cdot \hat{\mathbf{n}}=0
$$

where it is assumed that the surface current is negligible. ${ }^{28}$ The symbol $\|\xi\|$ stands for the jump of an arbitrary quantity $\xi$ across the interface. The current continuity condition also requires that the electric potential should not experience any jump at the interface, namely, $\|\phi\|=0 .{ }^{14,30}$ Finally, these interface conditions are automatically satisfied in the solution of relevant governing equations.

Dimensionless values are formed using the following scales:

$$
\begin{gathered}
\mathbf{x}=\mathbf{x}^{+} / r, \quad \rho=\rho^{+} / \rho_{d}, \quad \mu=\mu^{+} / \mu_{f} \quad \mathbf{u}=\mathbf{u}^{+} /\left(d / t^{+}\right), \\
t=t^{+} /\left(d /\left|\mathbf{u}_{c}\right|\right), \quad p=p^{+} / \rho_{d}\left(\left|\mathbf{u}_{c}\right|\right)^{2}, \\
\mathscr{D}=\rho_{d} / \rho_{f}, \quad \mathscr{V}=\mu_{d} / \mu_{f}, \quad \mathscr{P}=\varepsilon_{d} / \varepsilon_{f}, \quad \mathscr{C}=\sigma_{d} / \sigma_{f},
\end{gathered}
$$

leading to Reynolds, Weber, Electro-Weber, and electrical capillary numbers defined as

$$
\begin{gathered}
\operatorname{Re}=\frac{\rho_{d}\left|\mathbf{u}_{c}\right| d}{\mu_{d}}, \quad \mathrm{We}=\frac{\rho_{d}\left|\mathbf{u}_{c}\right|^{2} d}{\gamma}, \quad \mathrm{Ew}=\frac{\rho_{d}\left|\mathbf{u}_{c}\right|^{2}}{\varepsilon_{d} E_{\infty}^{2}}, \\
\mathrm{Ec}=\frac{\mathrm{We}}{\mathrm{Ew}}=\frac{\varepsilon_{d} E_{\infty}^{2} d}{\gamma} .
\end{gathered}
$$

Here, $\mathbf{u}_{c}=\varepsilon_{d} E_{\infty}^{2} d / \mu_{d}$ is the characteristic velocity, in which $d$ and $E_{\infty}$ are the droplet diameter and electric field intensity, respectively. Superscript + denotes dimensional variables, whereas subscripts $f$ and $d$ refer to background fluid and droplet phases, respectively.

\section{NUMERICAL METHOD}

Equations (1) and (2) are solved using the smoothed particle hydrodynamics (SPH) method. SPH is a mesh-free Lagrangian particle-based method, which was initially developed by Gingold and Monaghan $^{31}$ for astrophysical problems. However, it had been used for simulating a broad range of physical problems including free surface, ${ }^{32-34}$ turbulent, $^{35,36}$ multi-phase, ${ }^{15,37,38}$ heat transfer, ${ }^{39,40}$ and biological problems. ${ }^{41,42}$ In this section, the SPH method is described in more detail, which is used in our simulation.

To track the surface between the different phases, a color function $\hat{c}$ is defined. For each phase, a value of zero is assigned to one phase and unity for the other. To ensure a smooth transition between the phases and improve the accuracy of properties on the boundaries, at each time step, the initial color function is smoothed out across the phase boundaries using the following equation:

$$
c_{\mathrm{i}}=\sum_{\mathrm{j}=1}^{J_{n}} \frac{\hat{c}_{\mathrm{j}} W_{\mathrm{ij}}}{\psi_{\mathrm{i}}},
$$

where $\psi_{\mathrm{i}}=\sum_{\mathrm{j}=1}^{J_{n}} W_{\mathrm{ij}}$ is the particle number density, calculated as the sum of the interpolation kernel of neighboring particles $i$ and $j$ over all neighbors of particle $\mathrm{i}, J_{n} . W_{\mathrm{ij}}$ is a brief form of $W\left(r_{\mathrm{ij}}, h\right)$, the interpolation kernel, which is a function of $h$, the smoothing length, and the magnitude of distance vector, $\mathbf{r}_{i j}=\mathbf{r}_{i}-\mathbf{r}_{j}$, between the particle of interest $i$ and its neighboring particles $j .{ }^{43,44}$ In this study, the two-dimensional quintic spline kernel has been used. ${ }^{1}$

The smoothed color function is used to calculate the parameters in Eq. (4): $\delta \simeq|\nabla c|, \kappa=-\nabla \cdot \hat{\mathbf{n}}$, and $\hat{\mathbf{n}}=\nabla c /|\nabla c|$. To avoid the possible erroneous normals in this formulation, a constraint must be used. ${ }^{45}$ In this study, only gradient values exceeding a certain threshold, $\left|\nabla c_{\mathbf{i}}\right| \geq \alpha / h$, are used in surface tension force calculations, in which $\alpha$ is a constant value. In this study, $\alpha=0.08$ has been found to provide accurate results without removing too much detail. $^{46}$

Weighted Arithmetic Mean (WAM) is used to interpolate the phase properties, which is defined as

$$
\chi_{\mathrm{i}}=c_{\mathrm{i}} \chi_{d}+\left(1-c_{\mathrm{i}}\right) \chi_{f},
$$

in which $\chi$ denotes the hydrodynamic or electrical fluid properties, such as density, viscosity, permittivity, and conductivity.

To advance the governing equations of flow in time, the predictor-corrector scheme is used. The first-order Euler approach is carried out for the time discretization. To ensure the numerical convergence, the Courant-Friedrichs-Lewy (CFL) condition is used during the simulation to determine the variable time step size, $\Delta t$ $=\zeta h / u_{\max }$, where $u_{\max }$ is the largest particle velocity magnitude and $\zeta$ is taken to be equal to 0.25 . ${ }^{46}$ All the variables are advanced in time to the temporary or intermediate form using

$$
\begin{gathered}
\mathbf{r}_{\mathrm{i}}^{*}=\mathbf{r}_{\mathrm{i}}^{(n)}+\mathbf{u}_{\mathrm{i}}^{(n)} \Delta t+\delta \mathbf{r}_{\mathrm{i}}^{(n)}, \\
\mathbf{u}_{\mathrm{i}}^{*}=\mathbf{u}_{\mathrm{i}}^{(n)}+\frac{1}{\rho_{\mathrm{i}}^{(n)}}\left(\frac{1}{\operatorname{Re}} \nabla \cdot \boldsymbol{\tau}_{\mathrm{i}}+\frac{1}{\mathrm{We}} \mathbf{f}_{(s) \mathrm{i}}+\frac{1}{\mathrm{EW}} \mathbf{f}_{(e) \mathrm{i}}\right)^{(n)} \Delta t,
\end{gathered}
$$

$$
\psi_{\mathrm{i}}^{*}=\sum_{\mathrm{j}=1}^{J_{n}} W_{\mathrm{ij}}^{*} .
$$

Here, the starred variable represents an intermediate value and superscript $(n)$ denotes values at the $n$th time step. To prevent the particle clustering, the Artificial Particle Displacement (APD) method is used. In this method, the artificial particle displacement vector is implemented through $\delta \mathbf{r}_{\mathrm{i}}^{(n)}$ as

$$
\delta \mathbf{r}_{\mathrm{i}}^{(n)}=\beta\left[u_{\max } \sum_{\mathrm{j}=1}^{J_{n}}\left(\frac{\mathbf{r}_{\mathrm{ij}}}{r_{\mathrm{ij}}^{3}} r_{\mathrm{avg}, \mathrm{i}}^{2}\right)\right]^{(n)} \Delta t .
$$

Average particle spacing is found via $r_{\mathrm{avg}, \mathrm{i}}=\sum_{\mathrm{j}=1}^{J_{n}} r_{\mathrm{ij}} / J_{n}$ while a value of $\beta=0.06$ is employed as suggested in Ref. 37 to ensure a satisfactory particle distribution.

Using intermediate values, pressure at the next time step is found by solving the Poisson equation. To complete the temporal transition, the position and velocity of the particles are corrected, 


$$
\begin{gathered}
\nabla \cdot\left(\frac{1}{\rho_{\mathrm{i}}^{*}} \nabla p_{\mathrm{i}}^{(n+1)}\right)=\frac{\nabla \cdot \mathbf{u}_{\mathrm{i}}^{*}}{\Delta t}, \\
\mathbf{u}_{\mathrm{i}}^{(n+1)}=\mathbf{u}_{\mathrm{i}}^{*}-\frac{1}{\rho_{\mathrm{i}}^{*}} \nabla p_{\mathrm{i}}^{(n+1)} \Delta t, \\
\mathbf{r}_{\mathrm{i}}^{(n+1)}=\mathbf{r}_{\mathrm{i}}^{(n)}+\frac{1}{2}\left(\mathbf{u}_{\mathrm{i}}^{(n)}+\mathbf{u}_{\mathrm{i}}^{(n+1)}\right) \Delta t+\delta \mathbf{r}_{\mathrm{i}}^{(n)} .
\end{gathered}
$$

Boundary conditions are enforced through the multiple boundary tangent (MBT) method described in Ref. 47, while the first derivative and Laplace operator of vector functions are approximated through the following expressions:

$$
\begin{gathered}
\frac{\partial f_{\mathrm{i}}^{m}}{\partial x_{\mathrm{i}}^{k}} a_{\mathrm{i}}^{k l}=\sum_{\mathrm{j}} \frac{1}{\psi_{\mathrm{j}}}\left(f_{\mathrm{j}}^{m}-f_{\mathrm{i}}^{m}\right) \frac{\partial W_{\mathrm{ij}}}{\partial x_{\mathrm{i}}^{l}} \\
\frac{\partial}{\partial x_{\mathrm{i}}^{k}}\left(\varphi_{\mathrm{i}} \frac{\partial f_{\mathrm{i}}^{m}}{\partial x_{\mathrm{i}}^{k}}\right) a_{\mathrm{i}}^{m l}=8 \sum_{\mathrm{j}=1}^{J_{n}} \frac{2 \varphi_{\mathrm{i}} \varphi_{\mathrm{j}}}{\varphi_{\mathrm{i}}+\varphi_{\mathrm{j}}} \frac{1}{\psi_{\mathrm{j}}}\left(f_{\mathrm{i}}^{m}-f_{\mathrm{j}}^{m}\right) \frac{r_{\mathrm{ij}}^{m}}{r_{\mathrm{ij}}^{2}} \frac{\partial W_{\mathrm{ij}}}{\partial x_{\mathrm{i}}^{l}} .
\end{gathered}
$$

Here, $a_{\mathrm{i}}^{k l}=\sum_{\mathrm{j}} \frac{r_{\mathrm{ji}}^{k}}{\psi_{\mathrm{j}}} \frac{\partial W_{\mathrm{ij}}}{\partial x_{\mathrm{i}}^{l}}$ is a corrective second rank tensor that eliminates particle inconsistencies, ${ }^{48}$ while $\varphi$ may denote densityinversed, viscosity, permittivity, or conductivity, where appropriate. The left-hand sides of (18) and (7) as well as the right-hand side of (8) are discretized as

$$
\frac{\partial}{\partial x_{\mathrm{i}}^{k}}\left(\varphi_{\mathrm{i}} \frac{\partial f_{\mathrm{i}}}{\partial x_{\mathrm{i}}^{k}}\right)\left(2+a_{\mathrm{i}}^{k k}\right)=8 \sum_{\mathrm{j}=1}^{J_{n}} \frac{2 \varphi_{\mathrm{i}} \varphi_{\mathrm{j}}}{\varphi_{\mathrm{i}}+\varphi_{\mathrm{j}}} \frac{1}{\psi_{\mathrm{j}}}\left(f_{\mathrm{i}}-f_{\mathrm{j}}\right) \frac{r_{\mathrm{ij}}^{k}}{r_{\mathrm{ij}}^{2}} \frac{\partial W_{\mathrm{ij}}}{\partial x_{\mathrm{i}}^{k}} .
$$

\section{PROBLEM SETUP AND NUMERICAL CODE ACCURACY}

The computational domain is shown in Fig. 1, which consists of a 2D droplet with diameter $d$ suspended in an ambient fluid inside a confined square domain assuming $W=H$. The confinement ratio is defined as the ratio of the droplet diameter to the domain length, $W c=d / H$. A uniform electric field $E_{\infty}$ is applied in the downward direction. It is assumed that no gravitational force is applied and there is no relative motion between the droplet and ambient fluid initially. The no-slip boundary condition is applied on all side boundaries to imitate the confined domain. Considering the electric field, Dirichlet and Neumann boundary conditions are applied for horizontal and vertical walls, respectively. All particles inside and outside the droplet are arranged using a uniformly spaced Cartesian grid.

Considering the leaky dielectric model, the droplet may deform into two distinct configurations forming prolate or oblate shapes. The prolate shape is achieved when the droplet elongates in the direction of the applied electric field, while the transverse elongation of the droplet is known as oblate deformation. To check the dependency of numerical results with respect to the particle resolution, the deformation of the droplet in an unbounded domain, i.e., $W c=0$, is simulated for both prolate and oblate cases at $E c=0.02$. Fluid properties of the respective cases are tabulated in Table I. The simulations are performed for different values of particle resolution $x / d=20,40$, 60 , and 80 , where $x / d$ is the number of particles per unit of droplet's

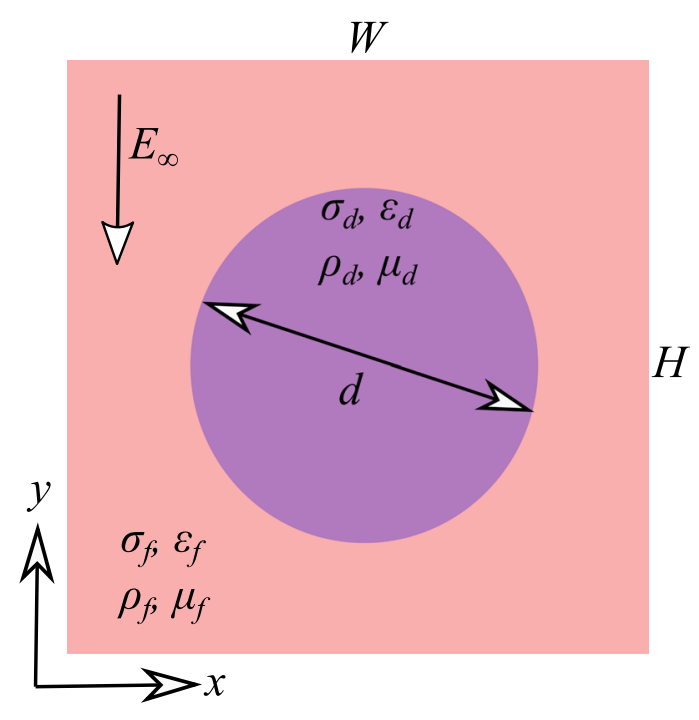

FIG. 1. Schematic representation of the physical setup including a suspended droplet with the initial diameter $d$ in an ambient fluid under the constant electric field applied in the downward direction.

initial diameter. The deformation of the droplet can be characterized as

$$
D=\frac{l_{1}-l_{2}}{l_{1}+l_{2}}
$$

where $l_{1}$ and $l_{2}$ are the two main axes of the deformed droplet in the directions parallel and perpendicular to the applied electric field, respectively. So, positive and negative values calculated by (24) represent prolate and oblate deformations, respectively. It should be noted that for the unbounded domain, $W c$ is considered to be small enough such that the effect of boundaries is negligible as shown in our previous studies (see, for example, Ref. 14).

The steady-state deformation of the droplet is represented in Fig. 2 for different resolutions. Qualitative and quantitative comparison of the results in Fig. 2 indicates that increasing the resolution from $x / d=20$ to 40 and from $x / d=40$ to 60 shows improvement in the accuracy of the results. However, no significant improvement has been observed from $x / d=60$ to 80 , so $x / d=60$ is considered as the reference particle resolution.

Our current in-house code has been validated in various cases including EHD simulation of a droplet, ${ }^{14}$ bubble rising, ${ }^{15}$ droplet coalescence and electro-coalescence, ${ }^{50}$ and other multiphase flow problems. ${ }^{37}$ However, further validation has been performed through simulating the droplet deformation under the effect of the electric field in an unbounded domain. It should be noted that the droplet in the absence of an electric field has also been

TABLE I. The ratio of the material properties, which is used in Sec. IV to prove the code accuracy and also resolution dependency test.

\begin{tabular}{lllll}
\hline \hline System & $\mathscr{P}$ & $\mathscr{C}$ & $\mathscr{D}$ & $\mathscr{V}$ \\
\hline Prolate & 0.5 & 2.0 & 1 & 1 \\
Oblate & 5.0 & 0.2 & 1 & 1 \\
\hline \hline
\end{tabular}



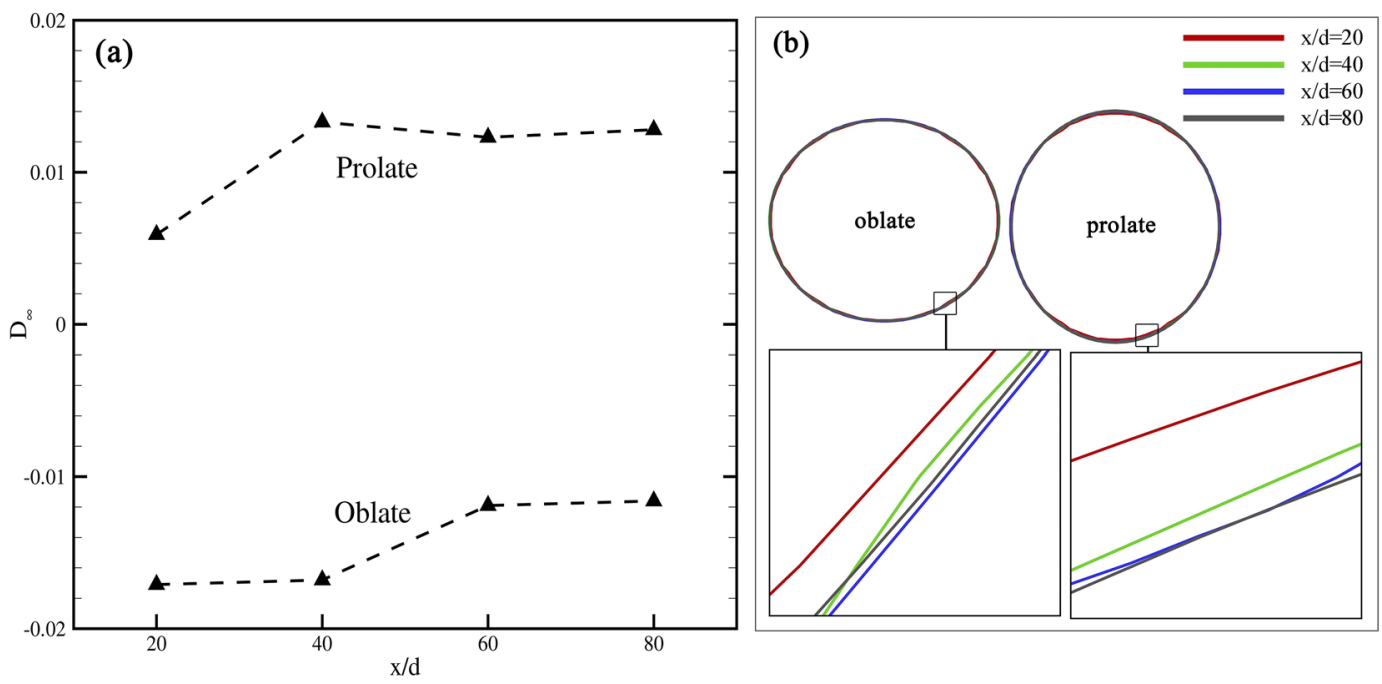

FIG. 2. Effect of the particle resolution on the droplet deformation in $W c=0$ and $E c=0.02$ : (a) quantitative result and (b) qualitative result.

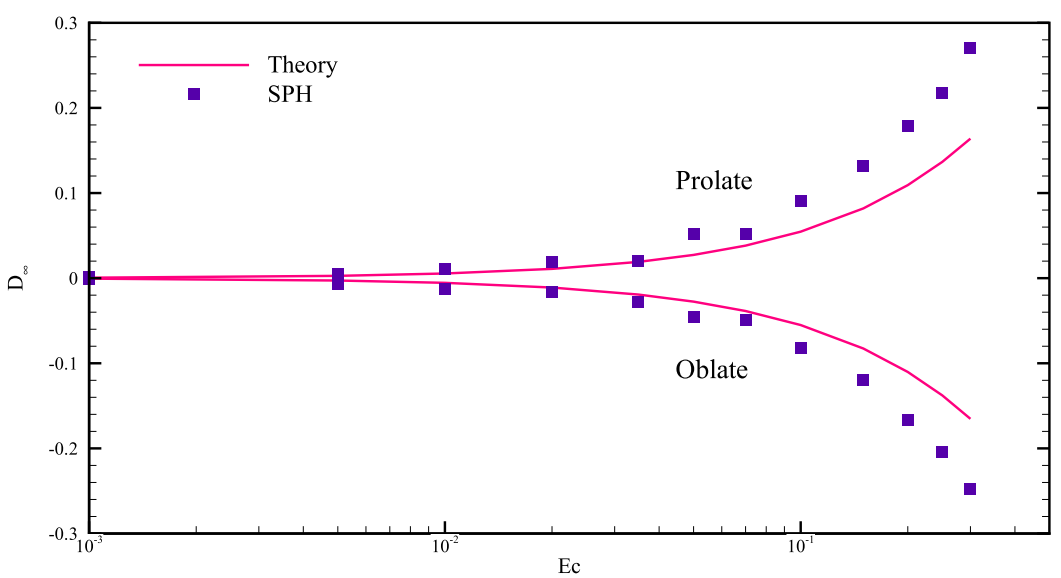

FIG. 3. The comparison of the droplet deformation between our numerical simulation and analytical data of Feng and Scott ${ }^{49}$ for $W c=0$ and different amounts of $E c$.

simulated in our previous study in which the pressure jump across the interface is validated with the Young-Laplace equation. To mitigate redundancy, we did not include those validations in this paper, but interested readers are referred to Ref. 14. In this section, only the deformation of a droplet in the presence of the electric field is being considered. To achieve this, two prolate and oblate systems based on the properties of Table I are modeled under various electrical capillary numbers $E c$. The effect of the electrical capillary number on the droplet deformation is shown in Fig. 3 and is compared with the analytical results of Feng and Scott. ${ }^{49}$ Feng and Scott used the following equation for calculating the deformation of the droplet as

$$
D=\frac{f_{d} E_{\infty}^{2} \varepsilon_{d}(d / 2)}{3(1+\mathscr{C})^{2} \mathscr{P} \gamma},
$$

where $f_{d}$ is the discriminating function defined as

$$
f_{d}=\mathscr{C}^{2}+\mathscr{C}+1-3 \mathscr{P} \text {. }
$$

As shown in Fig. 3, our results perfectly match with the analytical data for small deformations $\left(\left|D_{\infty}\right|<0.05\right)$. For larger deformations, however, it is observed that the numerical data deviate from the analytical data. This is due to the assumptions made in the theory, which considers the droplet to remain almost circular. Thus, the analytical predictions are only valid for small deformations. Such observations have been frequently reported by other numerical studies (see, for example, Refs. 8, 14, 51, and 52).

\section{NUMERICAL RESULTS}

In this section, the EHD deformation of a single droplet in a confined domain is presented by considering six different systems of fluids, as tabulated in Table II. The properties of the first four systems are selected from experiments in micro- and bio-fluidic applications. The properties of the other two systems are chosen hypothetically to illustrate all possible forms of droplet deformation, which will be thoroughly discussed. These fluid systems are selected 
TABLE II. Dielectric properties that are used in this study.

\begin{tabular}{|c|c|c|c|c|c|c|c|c|c|}
\hline System & Ambient fluid & $\sigma_{f}\left(\frac{S}{m}\right)$ & $\varepsilon_{f}\left(\frac{F}{m}\right)$ & Droplet fluid & $\sigma_{d}\left(\frac{S}{m}\right)$ & $\varepsilon_{d}\left(\frac{F}{m}\right)$ & $\mathscr{C}$ & $\mathscr{P}$ & References \\
\hline I & Silicon oil 1 & $2.67 \times 10^{-12}$ & 2.66 & Corn oil & $1.06 \times 10^{-11}$ & 3.24 & 3.9700 & 1.2180 & 53 \\
\hline II & Oxidized castor oil & $1.0 \times 10^{-9}$ & 6.3 & Silicone oil 5000 & $3.33 \times 10^{-11}$ & 2.77 & 0.0333 & 0.4397 & 6 \\
\hline III & Corn oil & $1.06 \times 10^{-11}$ & 3.24 & Silicon oil 1 & $2.67 \times 10^{-12}$ & 2.66 & 0.2519 & 0.8210 & 53 \\
\hline IV & Medium & $1.8 \times 10^{-2}$ & 80 & Cytoplasm & 0.5 & 75 & 27.7778 & 0.9375 & 54 \\
\hline V & Hypothetical & $\ldots$ & $\ldots$ & Hypothetical & $\ldots$ & $\ldots$ & 1.0 & 0.2 & $\ldots$ \\
\hline VI & Hypothetical & $\ldots$ & $\ldots$ & Hypothetical & $\ldots$ & $\ldots$ & 0.02 & 0.1 & $\ldots$ \\
\hline
\end{tabular}

such that they represent the electric conductivity of the droplet to be smaller (systems II and VI), in the same other (systems I, III, and V), and larger (system IV) than the ambient fluid. Since the deformation of droplets is limited to the confined characteristics of the domain, the electric capillary number is chosen such that the unbounded deformation of the respective systems is relatively small and equal to $\left|D_{\infty}\right|=0.05$, unless stated otherwise.

Figure 4 represents the deformation of the droplet normalized with respect to the unbounded deformation for the systems in Table II. In this figure, the present numerical data are further compared with the theoretical results of Behjatian and Esmaeeli. ${ }^{23}$ They have introduced an analytical solution for a confined droplet in circular domains by

$$
D=\frac{E c}{3} \frac{\Gamma^{2} \Phi}{(\mathscr{C}+1)^{2}},
$$

where

$$
\Gamma=\frac{\mathscr{C}+1}{(\mathscr{C}+1)-W c^{2}(\mathscr{C}-1)}
$$

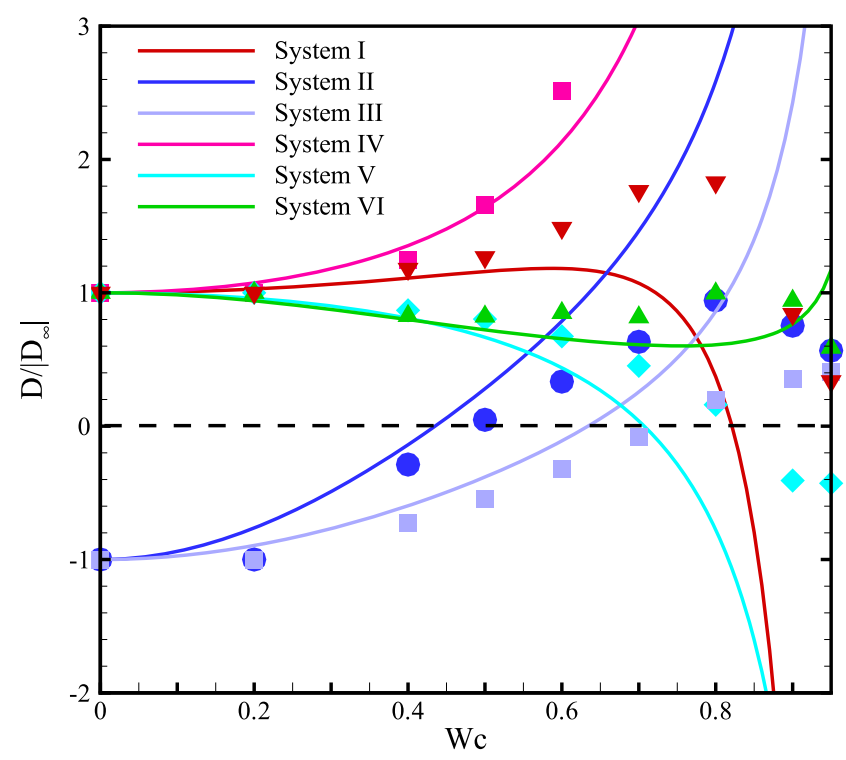

FIG. 4. Variation of deformation, which is normalized with the deformation at Wc $=0$ with respect to the confinement ratio. Solid lines demonstrate the analytical solution of Behjatian and Esmaeeli, ${ }^{23}$ and points represent the SPH results. and

$$
\Phi=\mathscr{C}^{2}+1-2 \mathscr{P}+\mathscr{F}(\mathscr{C}-\mathscr{P}),
$$

in which $\mathscr{F}$ is a characteristic function. Interested readers may refer to Ref. 23 for further information.

Equation (27) shows that for small confinement ratios (Wc $<0.4$ ), the normalized deformation does not represent significant variations. At larger confinement ratios, however, the deformation changes significantly in most cases. It is observed that for some of the systems, the droplet elongation shifts from oblate to prolate or vice versa. This behavior can be justified considering the variation in the magnitude and direction of electrical and hydrodynamic forces on the interface.

It is revealed that for small to moderate values of confinement ratio, i.e., $W c<0.6$, the present numerical method and the analytical solution produce similar results. However, our numerical results deviate from the theory at large confinement ratios. Considering the asymptotic behavior of the theoretical results for some of the presented systems, one can easily conclude that this cannot be valid due to the small and limited characteristic of a confined droplet deformation. Additionally, in some systems, the droplet might reach the boundaries and the corresponding deformation becomes undefined. The deformation of system IV, for instance, cannot be obtained beyond $W c=0.6$ since the droplet reaches the top and bottom boundaries. Thus, the analytical solution is only limited to small ranges of confinement ratio, while our SPH method can predict reasonable values for large confinement ratios, and corresponding values of deformation are limited and physically justifiable as shown in Fig. 4.

It can be shown that the hydrodynamics of the systems depends on the sign of $\mathscr{P}-\mathscr{C}$ such that the droplet and ambient fluids may circulate accordingly. ${ }^{14}$ So, systems V and II are chosen, which correspond to $\mathscr{P}<\mathscr{C}$ and $\mathscr{P}>\mathscr{C}$, respectively, to scrutinize the confinement effect on the characteristics of the flow. Figures 5(a) and 5 (c) demonstrate the streamlines for these systems at four different confinement ratios of $W c=0.6,0.7,0.8$, and 0.9. Due to the symmetric nature of the problem in $x$ and $y$ directions, only a quarter of the domain is shown. In each quarter, the flow consists of a pair of vortices inside and outside of the droplet, circulating in opposite directions since they match with their counterpart. For system II, outside velocities run from the left and the right $(\theta=0$ and $\pi)$ toward the top and the bottom $(\theta=\pi / 2$ and $3 \pi / 2)$, which leads to the droplet elongation in the $x$ direction (oblate shape) in an unbounded domain. For system $\mathrm{V}$, ambient velocities run from the top and the bottom $(\theta=\pi / 2$ and $3 \pi / 2)$ toward the left and the right $(\theta=0$ 
(a)

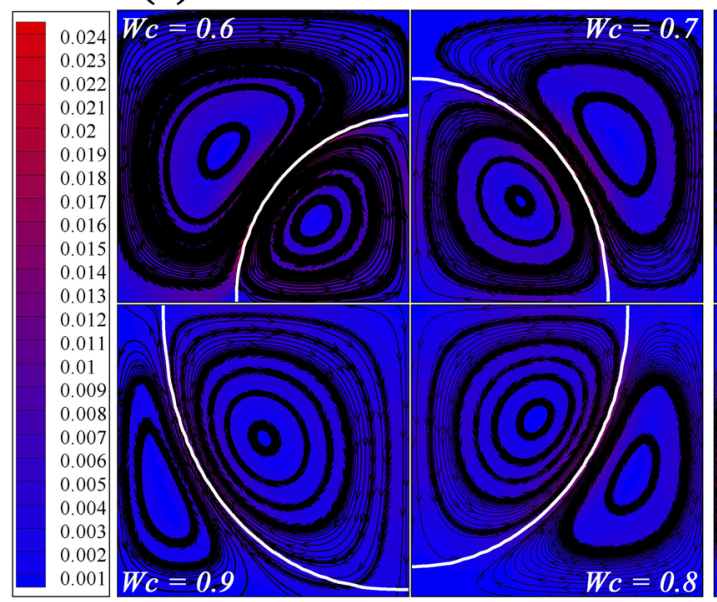

(b)

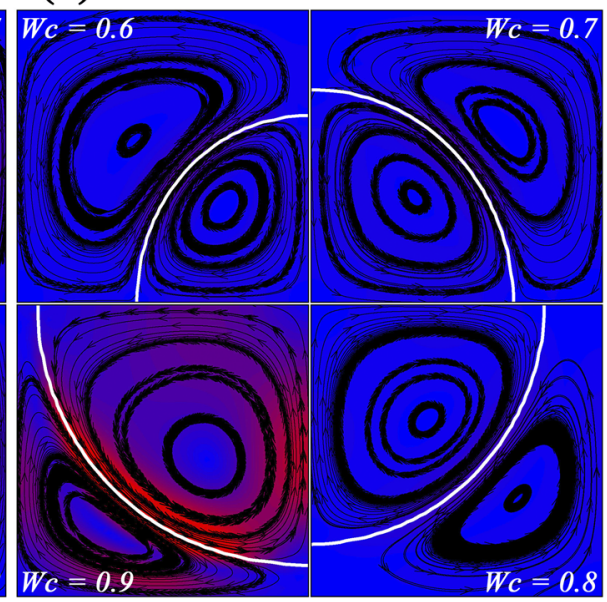

FIG. 5. Streamlines and magnitude of velocity contours for (a) system II and (b) system V at various values of Wc. Due to symmetry in $x$ and $y$ directions, only a quarter of the domain is shown.

and $\pi$ ) as shown in Fig. 5, resulting in elongation of the droplet in the $y$ direction (prolate shape) at small confinement ratios. At large confinement ratios, however, the deformation of these two systems shifts from prolate to oblate and vice versa. Nevertheless, by increasing the confinement ratio, the circulation direction of the vortices in the interior and exterior regions is not changed. Thus, the change in the deformation due to the confinement ratio variation cannot be justified by considering the vortex structure only. Therefore, it is needed to analyze the electrical and hydrodynamic forces on the interface of the droplet to investigate the reason for the deformation variation, precisely.

As mentioned in Sec. I, Santra et al. investigated the effect of the confinement ratio on the magnitude of velocity on a line passing through the center of the droplet. ${ }^{9}$ To see the effect of the confinement ratio on the velocity magnitude of the entire domain, contours of this parameter are calculated and plotted in Figs. 5(a) and 5(b) for systems II and V, respectively, at various amounts of confinement ratios. As shown in this figure, by increasing the confinement ratio for system II, the velocity magnitude reacts inversely and its value decreases inside the domain, which is consistent with the results of Santra et $a .^{9}$ In contrast to system II, the velocity magnitude increases by increasing the confinement ratio for system $\mathrm{V}$ as shown in Fig. 5(b). As demonstrated in this figure, the velocity magnitude is relatively high at the top and bottom sides of the droplet at $W c=0.9$, which can prove the reason for the oblate deformation of system $\mathrm{V}$ at high confinement ratios. However, a detailed investigation of the competing interfacial forces is needed, which will be provided in the following.

Figure 6 represents the polarization (a), Coulomb (b), and total (c) electric forces by showing their direction and magnitude on the droplet interface for systems I, III, and V at $W c=0.9$. These three systems are selected since they represent different behavior due to different electric force configurations. It can be easily concluded from Eq. (5) that the polarization force acts normal to the interface in the opposite direction of the electrical permittivity gradient, while the direction of the Coulomb force depends on the interplay between the electric field and the electrical surface charges. Considering Fig. 6(a), the polarization force stretches the droplet into a prolate shape by acting at the top and bottom poles of the droplet in system I. In system III, the effect of the polarization force is more pronounced at the side of the droplet direction inward, while system V shows an almost constant distribution of the polarization force across the interface toward the center of the droplet. Figure 6(b) shows that the Coulomb force acts mainly parallel to the applied electric field vertically, representing an outward direction for systems I and V, in contrast to system III. By comparing the magnitude of the polarization and Coulomb force terms, Fig. 6(c) shows the effect of the total electric force for these three systems. For system I, the Coulomb and polarization forces are in the same direction acting at the top and bottom poles, magnifying their effect. For system III, the Coulomb force is much stronger than the polarization force, and thus, it dominates the total electric force influence. For system $\mathrm{V}$, the polarization and Coulomb forces act in opposite directions especially at the top and bottom parts of the droplet. The interplay between these two terms results in an inward contribution from the polarization force at the side of the droplet and an outward effect at the top and bottom due to the stronger Coulomb force. It should be noted that the electric force configurations of systems II and VI are the same as III, so they are not represented here for the sake of brevity. Additionally, the polarization and Coulomb forces act on the top and bottom poles toward and outward the center, respectively, for system IV, while the magnitude of the Coulomb force is greater than the polarization force. Hence, the total electric force stretches the droplet in a vertical direction similar to system I, as shown in Figs. 7(a) and $7(\mathrm{c})$.

Recalling from Fig. 4, $\left|D_{\infty}\right|$ is set to 0.05 for all systems and the $D /\left|D_{\infty}\right|$ is evaluated up to $W c=0.95$. On the other hand, it can be observed from the analytical results shown in Fig. 4 that systems II and IV tend to reach very large deformations at high confinement ratios (i.e., $W c>0.7$ ). However, we observe that systems II and IV 

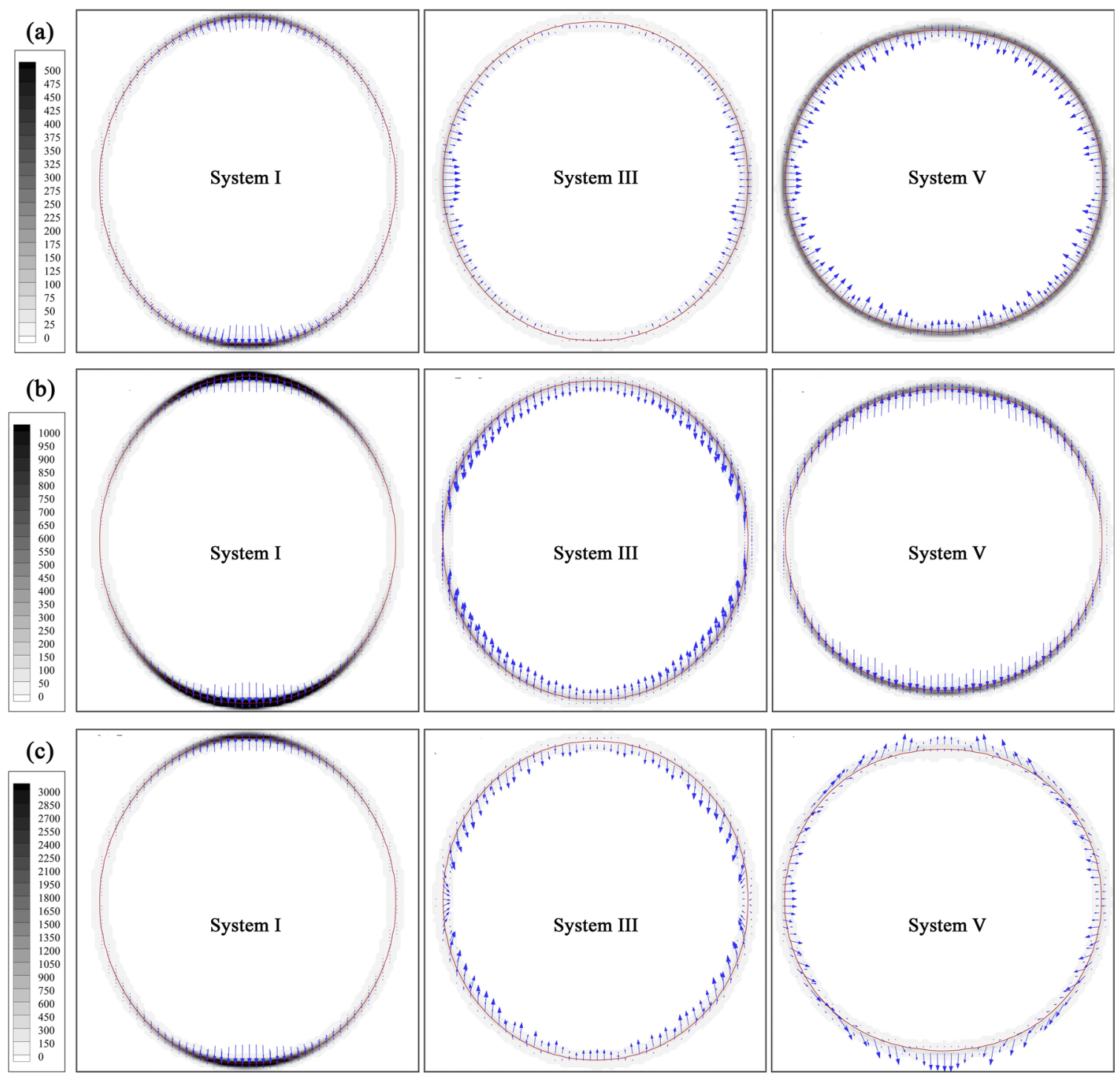

FIG. 6. Comparison of (a) polarization force, (b) Coulomb force, and (c) total electric force on the interface of the droplet at Wc $=0.9$ for systems I, III, and V.
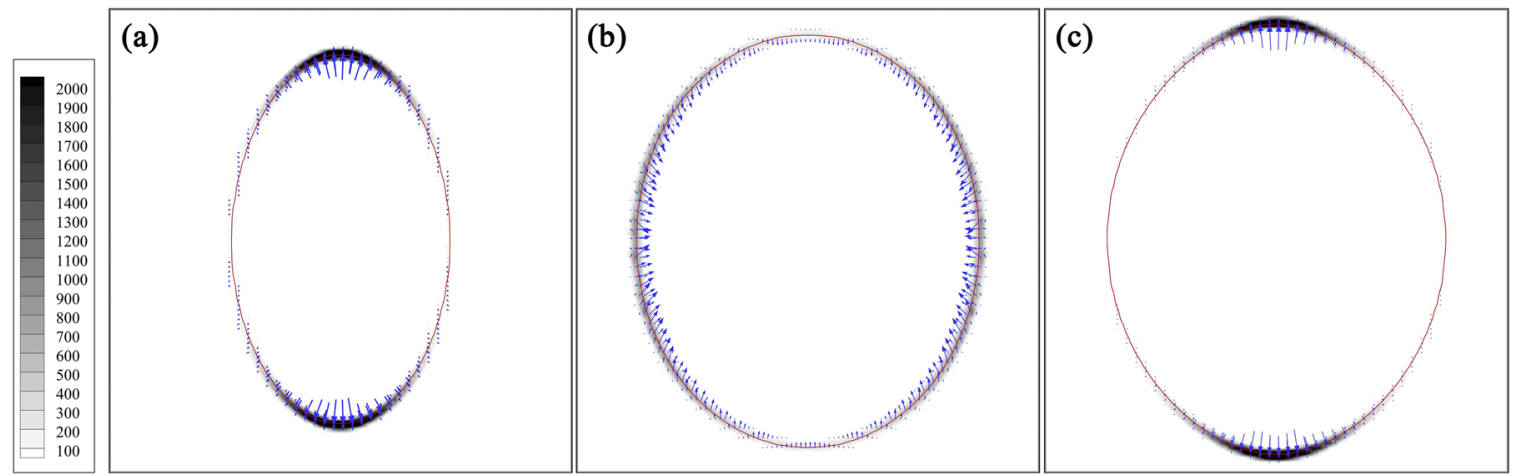

FIG. 7. Total electric force on the interface of the droplet for (a) system IV at $W c=0.6$ and $\left|D_{\infty}\right|=0.05$, (b) system II at $W c=0.8$ and $\left|D_{\infty}\right|=0.05$, and (c) system IV at $W c$ $=0.8$ and $\left|D_{\infty}\right|=0.01$. 
behave differently under the EHD effects. The discrepancy is due to different electric force field configurations on the droplet interface. Figure 7 represents the deformation of systems II and IV and illustrates the electric force vectors and their magnitude on the interface. It can be clearly seen in Fig. 7(a) that the electric forces act at the top and bottom poles of the droplet in system IV and elongate it into a prolate shape, while the cause for the deformation of system II is the inward electric forces at the side poles of the droplet as shown in Fig. 7(b). Additionally, the magnitude of the electric forces is much larger in system IV. This results in large droplet deformations at relatively smaller confinement ratios where the droplet reaches the top and bottom boundaries at $W c>0.6$. In order to represent the deformation of system IV at larger confinement ratios, the unbounded deformation has been changed from $\left|D_{\infty}\right|=0.05$ to $\left|D_{\infty}\right|=0.01$, which corresponds to the smaller electrical capillary number. Hence, this indicates that the applied electric force is weaker on the droplet interface leading to smaller unbounded deformations. Under this condition, the droplet deformation of system IV can be tracked up to large confinement ratios i.e., $W c=0.8$ reaching $D /\left|D_{\infty}\right|$ $=6.96$ as represented in Fig. 7(c). This shows that the analytical solution predicts the deformation of system IV correctly only at very small unbounded deformations. For system II, however, the analytical solution does not represent accurate results at large confinement ratios.

In Fig. 7 , it is shown that the value of the unbounded deformation (e.g., in system IV) affects the predictions at large confinement ratios and results in different $\mathrm{D} /\left|\mathrm{D}_{\infty}\right|$. In order to analyze this effect, Fig. 8 represents $\mathrm{D} /\left|\mathrm{D}_{\infty}\right|$ at two confinement ratios (a) Wc $=0.6$ and (b) $W c=0.8$ for all six systems in Table II as a function of $\left|D_{\infty}\right|$, which can be interpreted as an effect of the applied electric field strength. At $W c=0.6$, it is observed that the analytical solution provides relatively accurate results for all systems. For systems II and V, the variation of the unbounded deformation does not show a considerable effect on $\mathrm{D} /\left|\mathrm{D}_{\infty}\right|$, while it is observed that for systems III and VI, the analytical solution predicts more accurate results at large unbounded deformations. On the other hand, the variations of systems I and IV do not indicate a meaningful relationship. At $W c=0.8$, systems I and VI represent an approaching trend as the unbounded deformation increases, in contrast to system II, while systems III and V do not show remarkable sensitivity to the variations of unbounded deformation. For system IV, there are no available data at this confinement due to the extreme action of the electric forces by which the droplet reaches the top and bottom boundaries at very small unbounded deformations (i.e., $\left.\mathrm{D}_{\infty}<0.02\right)$. By analyzing the data, one may notice that the analytical solution is accurate at small and moderate confinement ratios $(W c \leq 0.6)$, which confirms our previous conclusion. For high confinement ratios, on the other hand, high unbounded deformation should be selected for making analytical solutions applicable. Otherwise, there will be a difference between analytical and numerical results. The reason behind such a difference is due to the existence of complex interactions and the interplay between electrical and hydrodynamic forces that we aim to elaborate for some of the systems in the following.

Recalling from Fig. 4, one can notice that the general trend of systems I and V is similar except the difference in numerical results at large confinement ratios. It should be noted that for both systems $\mathrm{I}$ and $\mathrm{V}$, the comparison of the electrical conductivity and permittivity ratios yields $\mathscr{P}<\mathscr{C}$. So, the inner and outer vortices around the droplet are as described in Fig. 5(b). Additionally, the electric forces on the interface tend to deform the droplet into a prolate shape as shown in Figs. 6(a)-6(c), as observed for both cases at small confinement ratios. In contrast, the electrical conductivity and permittivity ratios of systems II and III result in $\mathscr{P}>\mathscr{C}$, so it is expected that they deform into an oblate shape as seen in small confinement ratios. However, the deformations of these two systems represent a (a)

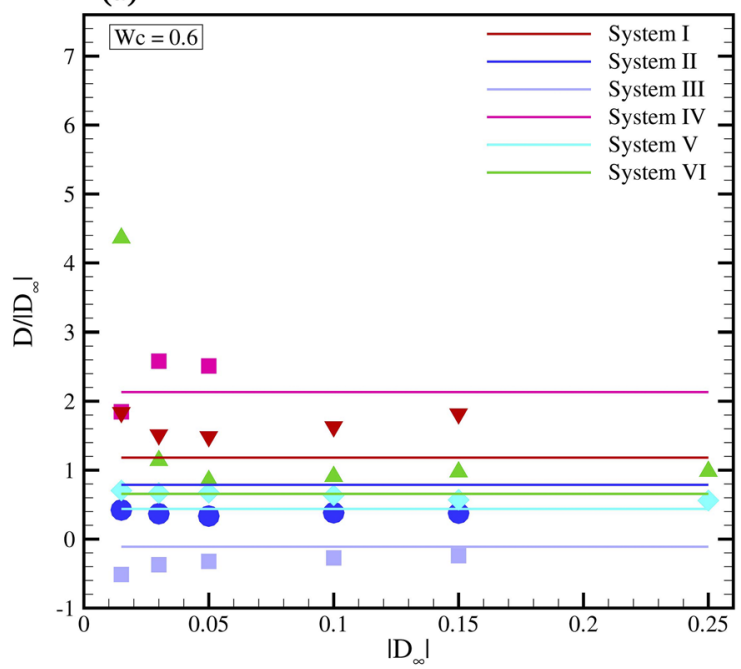

(b)

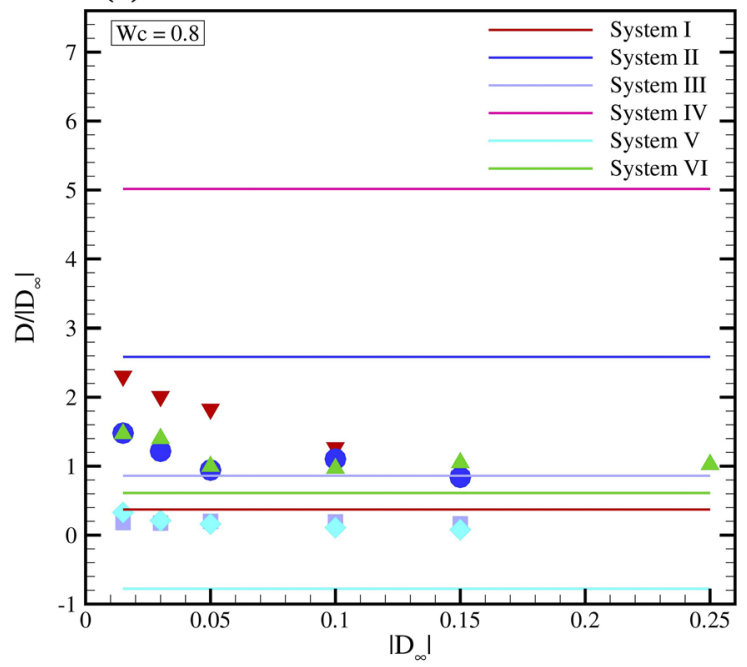

FIG. 8. Effect of unbounded deformation on the variation of normalized droplet deformation at (a) $W c=0.6$ and (b) $W c=0.8$. Solid lines demonstrate the results of Behjatian and Esmaeeli, ${ }^{23}$ and points represent the SPH results. 
shift from oblate to prolate deformation at large confinement ratios. In order to explain the above-mentioned cases, other dominating terms, such as pressure, should also be considered, which can highly influence the hydrodynamics of microfluidic systems. We believe that the reason for such discrepancies is inherited in inappropriate utilization of the pressure term in the analytical solution. In the following, it will be shown how the pressure dominates the flow and changes the hydrodynamics of the system. This will be represented in Fig. 9 by comparing the dynamics of systems I and V, as well as in Fig. 10 for systems II and III.
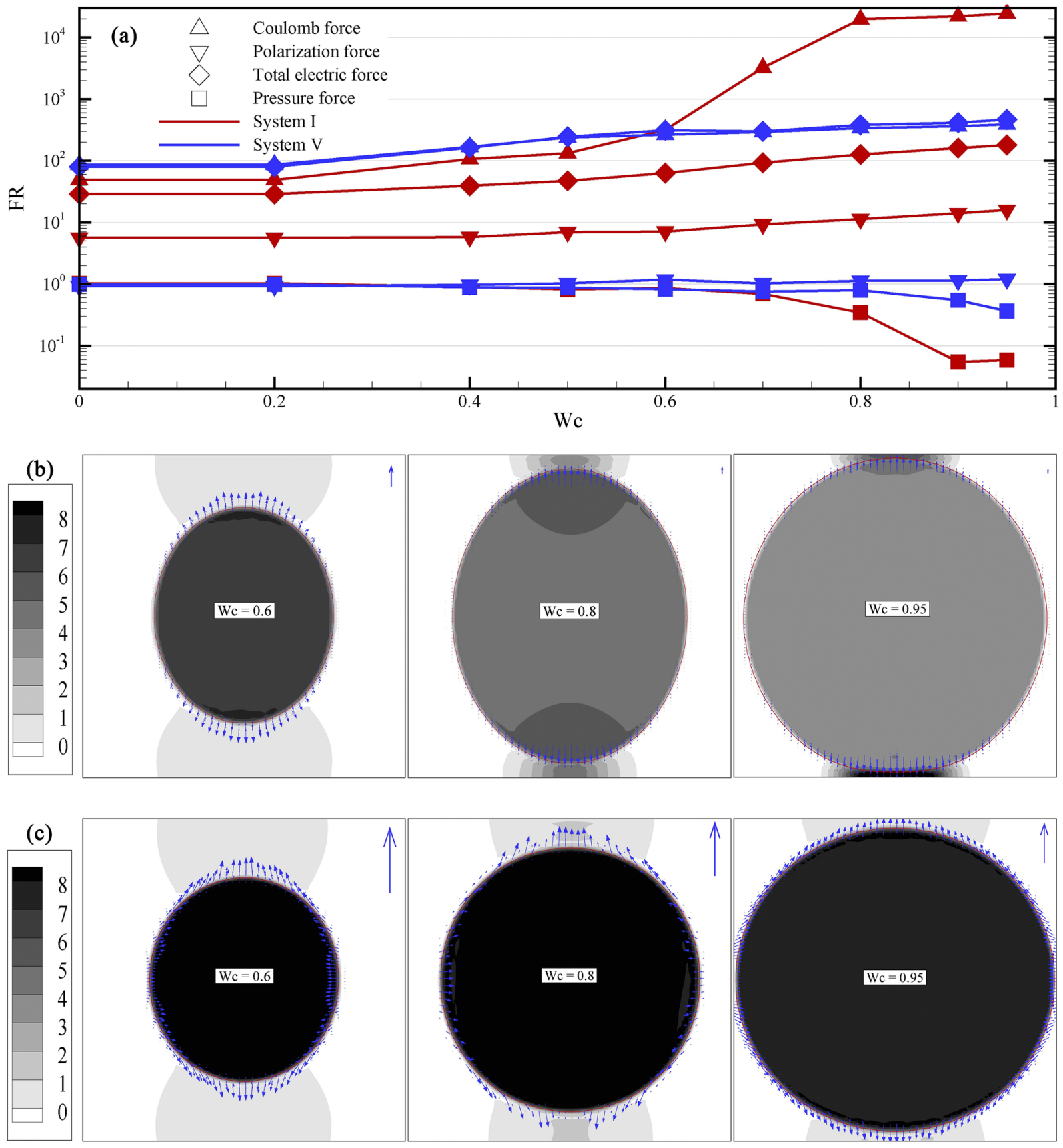

FIG. 9. Comparison between forces on the interface of systems I and V. (a) Effect of the confinement ratio on the force ratio of points on the vertical and horizontal axes of the droplet, which act in the prolate elongation. Pressure contour and vectors of the total electric force for (b) system I and (c) system V. Unit arrows are shown at the top-right corner of the sub-figures. 

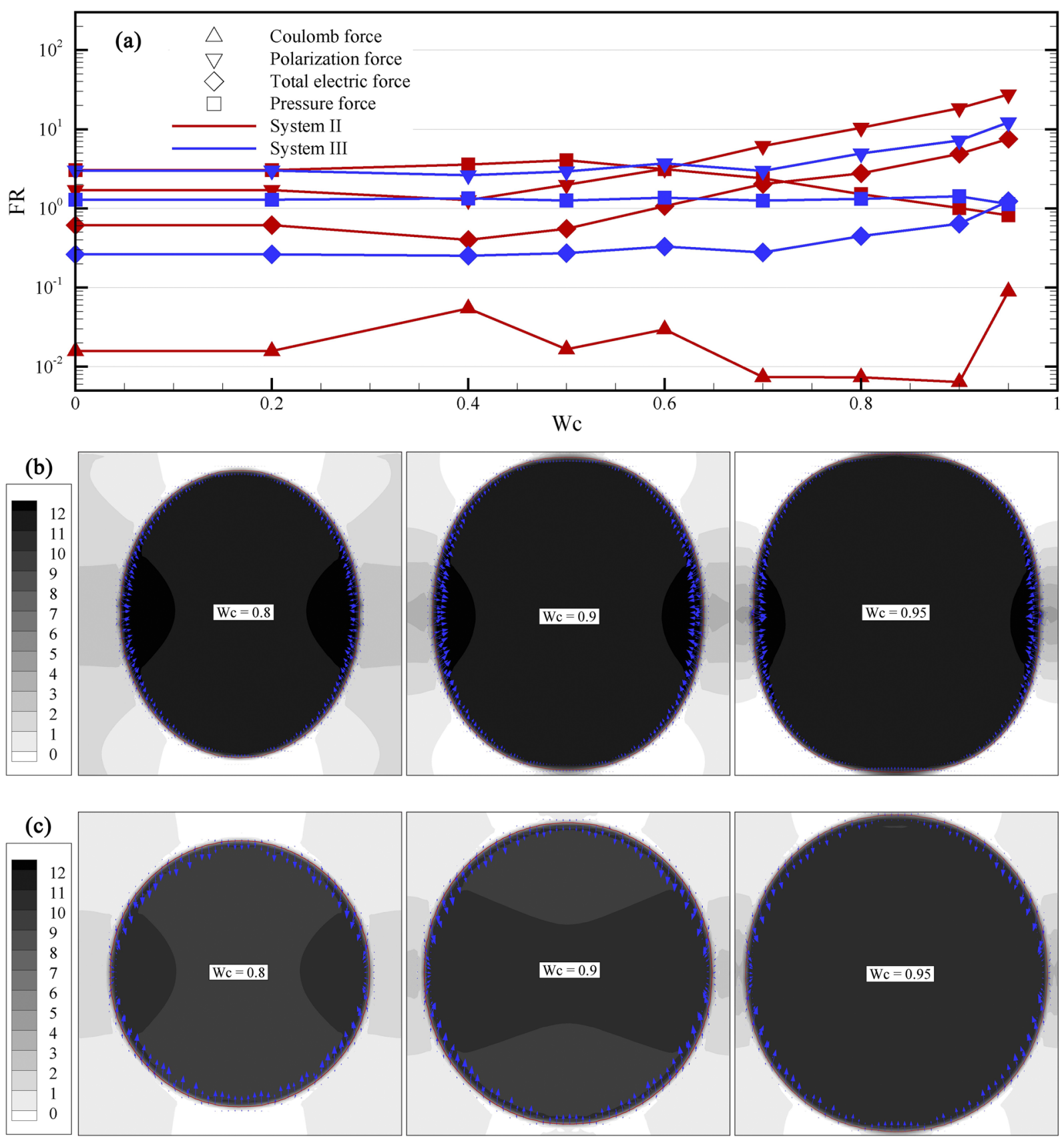

FIG. 10. Comparison between forces on the interface of systems II and III. (a) Effect of the confinement ratio on the force ratio of points on the vertical and horizontal axes of the droplet, which act in the prolate elongation. Pressure contour and vectors of the total electric force for (b) system II and (c) system III.

Figure 9 compares the electrical and pressure forces for systems I and V. In Fig. 9(a), a new parameter is introduced here, namely, the Force Ratio $(F R)$, which is the ratio of forces normal to the interface at the sides and top/bottom poles of the droplet. The $F R$ is defined such that if $F R>1$, interfacial forces deform the droplet into a prolate shape, while $F R<1$ leads to an oblate elongation. Figures 9 (b) and 9 (c) represent the contours of the pressure and the electric force vectors of systems $\mathrm{I}$ and $\mathrm{V}$ at $W c=0.6,0.8$, and 0.95 , respectively. It is observed that system I remains prolate at all confinement ratios, while system $\mathrm{V}$ shifts in an oblate deformation at large confinement ratios. Considering FR of system I, both polarization and Coulomb forces increase at large confinement 
ratios, while this increment is significant for the Coulomb force, resulting in the growth of the total electric force on the interface. Accordingly, one can expect the monotonical increase of the prolate deformation with respect to the confinement ratio. As shown in Fig. 4, however, the deformation value starts to decrease at $W c$ $=0.8$. On the other hand, $F R$ of the pressure indicates that at small confinement ratios, this value is around unity, meaning that the pressure is distributed equally around the interface. However, by further increasing the confinement ratio above $W c>0.7$, FR decreases such that the pressure force pushes the droplet from top/bottom poles of the droplet, so it acts in the opposite direction of the electric forces. At very large confinement ratios, i.e., $W c>0.8$, the pressure dominates the electric forces leading to a reduction in the prolate deformation of the droplet in system I. This explains the maximum prolate deformation at $W c=0.8$. For system $V$, the $F R$ of the polarization force remains almost constant across all confinement ratios, while the Coulomb force slightly increases at large confinement ratios. On the other hand, the pressure FR decreases such that it dominates the Coulomb force. As can be seen in Figs. 9(b) and $9(\mathrm{c})$, the magnitude of the electric forces in system I is much larger than those in system $\mathrm{V}$ as represented by the unit arrows shown at the top-right corner of the sub-figures. So, the decrease of the pressure $F R$ is magnified enough at very large confinement ratios so that the droplet exhibits an oblate deformation as shown in Fig. 4.

A similar analysis has been conducted here for systems II and III as demonstrated in Fig. 10. In contrast to systems I and V, systems II and III have oblate deformations at small confinement ratios, but increasing the confinement ratio will change their deformation into prolate shapes. By analyzing the force ratio FR of both systems in Fig. 10(a), it is revealed that the Coulomb force ratio is very small at small confinement ratios, showing an extreme tendency to deform the droplet into an oblate shape. It should be noted that the Coulomb force ratio of system III is extremely small, i.e., $O\left(10^{-4}\right)$, and thus, it is not shown in Fig. 10(a). The polarization force ratio, on the other hand, is in the order of $O\left(10^{\circ}\right)$ for both cases. This results in a total electric force ratio in the range of $O\left(10^{-1}\right)$, which induces oblate deformation for both systems, despite the presence of the opposing pressure force ratio. Subsequently, the interplay between the resultant electric force and the pressure enforces the droplet to deform into an oblate shape. By increasing the confinement ratio, the Coulomb $F R$ remains in the same order of magnitude, while the polarization force ratio monotonically increases for both systems. So, the resultant electric force has an increasing trend that changes the deformation of both systems from oblate to prolate elongation. It is observed for system II that the pressure force ratio decreases considerably such that it balances the effect of the total electric force in the opposite way. This reduces the prolate deformation of the droplet in system II at very large confinement ratios $W c>0.8$. This condition is not observed in system III.

\section{CONCLUSION}

In this paper, the EHD behavior of a droplet is studied in a highly confined domain by using the multi-phase ISPH method. Six different fluid systems are selected corresponding to the different electrical properties. It was shown that in the unbounded domain, ratios of the electrical properties are the main factors in determining the droplet deformation shape and values. By increasing the confinement ratios, the droplet deformation value was changed and in some cases, switched from oblate/prolate shape to prolate/oblate elongation. The dependency of the droplet deformation on the electric force components, including polarization and Coulomb forces, is discussed thoroughly. It was shown that these forces are the main reason for the droplet deformation and their strengths are highly dependent on the confinement ratio. The effect of the pressure force is also considered, and it was shown that its contribution becomes significant at high confinement ratios where it acts in the opposite direction with the electric force, decreasing the deformation value at high confinement ratios. To simplify the analysis, the force ratio was defined and used to show the contribution of each effective force on the droplet deformation. These force ratios are then sketched as a function of confinement ratio for some systems to better understand their effect and simplify their comparison.

\section{DATA AVAILABILITY}

The data that support the findings of this study are available from the corresponding author upon reasonable request.

\section{REFERENCES}

${ }^{1}$ Q. Sun, D. Wang, Y. Li, J. Zhang, S. Ye, J. Cui, L. Chen, Z. Wang, H.-J. Butt, D. Vollmer, and X. Deng, "Surface charge printing for programmed droplet transport," Nat. Mater. 18(9), 936-941 (2019).

${ }^{2}$ Y. Xia, "Electrohydrodynamic droplet injection method into model oil," AIP Adv. 9(10), 105309 (2019).

${ }^{3}$ K. Mohammadi, M. R. Movahhedy, and S. Khodaygan, "A multiphysics model for analysis of droplet formation in electrohydrodynamic 3D printing process," J. Aerosol Sci. 135, 72-85 (2019).

${ }^{4}$ E. Altun, N. Ekren, S. E. Kuruca, and O. Gunduz, "Cell studies on electrohydrodynamic (EHD)-3D-bioprinted bacterial cellulose \polycaprolactone scaffolds for tissue engineering," Mater. Lett. 234, 163-167 (2019).

${ }^{5}$ G. I. Taylor, "Disintegration of water drops in an electric field," Proc. R. Soc. London, Ser. A 280(1382), 383-397 (1964).

${ }^{6}$ R. S. Allan, S. G. Mason, and L. E. Marion, "Particle behaviour in shear and electric fields I. Deformation and burst of fluid drops," Proc. R. Soc. London, Ser. A 267(1328), 45-61 (1962).

${ }^{7}$ G. I. Taylor, A. D. McEwan, and L. N. J. de Jong, "Studies in electrohydrodynamics. I. The circulation produced in a drop by an electric field," Proc. R. Soc. London, Ser. A 291(1425), 159-166 (1966).

${ }^{8}$ I. Roghair, M. Musterd, D. van den Ende, C. Kleijn, M. Kreutzer, and F. Mugele, "A numerical technique to simulate display pixels based on electrowetting," Microfluid. Nanofluid. 19(2), 465-482 (2015).

${ }^{9}$ S. Santra, S. Mandal, and S. Chakraborty, "Electrohydrodynamics of confined two-dimensional liquid droplets in uniform electric field," Phys. Fluids 30(6), 062003 (2018).

${ }^{10}$ A. L. Kupershtokh and D. A. Medvedev, "Lattice Boltzmann equation method in electrohydrodynamic problems," in Fifth International Electrohydrodynamics (EHD) Workshop and Fourth Conference of the Société Française d'Electrostatique (SFE) [J. Electrostat. 64(7), 581-585 (2006)].

${ }^{11}$ M. Lauricella, S. Melchionna, A. Montessori, D. Pisignano, G. Pontrelli, and S. Succi, "Entropic lattice Boltzmann model for charged leaky dielectric multiphase fluids in electrified jets," Phys. Rev. E 97, 033308 (2018).

${ }^{12}$ E. Lac and G. M. Homsy, "Axisymmetric deformation and stability of a viscous drop in a steady electric field," J. Fluid Mech. 590, 239-264 (2007).

${ }^{13}$ M. N. Reddy and A. Esmaeeli, "The EHD-driven fluid flow and deformation of a liquid jet by a transverse electric field," Int. J. Multiphase Flow 35(11), 1051-1065 (2009). 
${ }^{14}$ M. S. Shadloo, A. Rahmat, and M. Yildiz, "A smoothed particle hydrodynamics study on the electrohydrodynamic deformation of a droplet suspended in a neutrally buoyant Newtonian fluid," Comput. Mech. 52(3), 693-707 (Sep 2013).

${ }^{15}$ A. Rahmat, N. Tofighi, and M. Yildiz, "Numerical simulation of the electrohydrodynamic effects on bubble rising using the SPH method," Int. J. Heat Fluid Flow 62, 313-323 (2016).

${ }^{16}$ J.-J. Xu, W. Shi, W.-F. Hu, and J.-J. Huang, "A level-set immersed interface method for simulating the electrohydrodynamics," J. Comput. Phys. 400, 108956 (2020).

${ }^{17}$ G. Fink, H. Medina, A. Springer, R. Wille, and H. Werner, "Design and realization of flexible droplet-based lab-on-a-chip devices," Elektrotech. Informationstech 137, 113 (2020).

${ }^{18}$ J. Liu, L. Tian, Y. Qiao, S. Zhou, A. J. Patil, K. Wang, M. Li, and S. Mann, "Hydrogel-immobilized coacervate droplets as modular microreactor assemblies," Angew. Chem., Int. Ed. 59(17), 6853-6859 (2020).

${ }^{19}$ W. Cui, G. Yesiloz, and C. L. Ren, "Numerical analysis on droplet mixing induced by microwave heating: Decoupling of influencing physical properties," Chem. Eng. Sci. 224, 115791 (2020).

${ }^{20} \mathrm{~S}$. Chakraborty and R. Mittal, "Droplet dynamics in a microchannel subjected to electrocapillary actuation,” J. Appl. Phys. 101(10), 104901 (2007).

${ }^{21}$ Y. Zhu and Q. Fang, "Analytical detection techniques for droplet microfluidicsA review," Anal. Chim. Acta 787, 24-35 (2013).

${ }^{22}$ J. M. Köhler, T. Henkel, A. Grodrian, T. Kirner, M. Roth, K. Martin, and J. Metze, "Digital reaction technology by micro segmented flow-components, concepts and applications," in 7th International Conference on Microreaction Technology [Chem. Eng. J. 101(1), 201-216 (2004)].

${ }^{23}$ A. Behjatian and A. Esmaeeli, "Electrohydrodynamics of a liquid column under a transverse electric field in confined domains," Int. J. Multiphase Flow 48, 71-81 (2013).

${ }^{24}$ A. Esmaeeli and A. Behjatian, "Electrohydrodynamics of a liquid drop in confined domains," Phys. Rev. E 86, 036310 (2012).

${ }^{25}$ S. Santra, S. Das, and S. Chakraborty, "Electrically modulated dynamics of a compound droplet in a confined microfluidic environment," J. Fluid Mech. 882, A23 (2020).

${ }^{26}$ A. Esmaeeli and A. Behjatian, "Transient electrohydrodynamics of a liquid drop at finite Reynolds numbers," J. Fluid Mech. 893, A26 (2020).

${ }^{27}$ J. U. Brackbill, D. B. Kothe, and C. Zemach, "A continuum method for modeling surface tension,” J. Comput. Phys. 100(2), 335-354 (1992).

${ }^{28}$ D. A. Saville, "Electrohydrodynamics: The Taylor-Melcher leaky dielectric model," Annu. Rev. Fluid Mech. 29(1), 27-64 (1997).

${ }^{29}$ J. Hua, L. K. Lim, and C.-H. Wang, "Numerical simulation of deformation/motion of a drop suspended in viscous liquids under influence of steady electric fields," Phys. Fluids 20(11), 113302 (2008).

${ }^{30}$ J. M. López-Herrera, S. Popinet, and M. A. Herrada, "A charge-conservative approach for simulating electrohydrodynamic two-phase flows using volume-offluid," J. Comput. Phys. 230(5), 1939-1955 (2011).

${ }^{31}$ R. A. Gingold and J. J. Monaghan, "Smoothed particle hydrodynamics: Theory and application to non-spherical stars," Mon. Not. R. Astron. Soc. 181(3), 375-389 (1977).

${ }^{32}$ R. Saghatchi, J. Ghazanfarian, and M. Gorji-Bandpy, "Numerical simulation of water-entry and sedimentation of an elliptic cylinder using smoothedparticle hydrodynamics method," J. Offshore Mech. Arct. Eng. 136(3), 031801 (2014).

${ }^{33}$ M. Ozbulut, N. Tofighi, O. Goren, and M. Yildiz, "Investigation of wave characteristics in oscillatory motion of partially filled rectangular tanks," J. Fluids Eng. 140(4), 041204 (2017).
${ }^{34}$ M. Ozbulut, S. Ramezanzadeh, M. Yildiz, and O. Goren, "Modelling of wave generation in a numerical tank by SPH method," J. Ocean Eng. Mar. Energy 6, 2198-6452 (2020).

${ }^{35}$ J. Ghazanfarian, R. Saghatchi, and M. Gorji-Bandpy, "Turbulent fluid-structure interaction of water-entry/exit of a rotating circular cylinder using SPH method," Int. J. Mod. Phys. C 26(08), 1550088 (2015).

${ }^{36}$ J. Ghazanfarian, R. Saghatchi, and M. Gorji-Bandpy, "Sph simulation of turbulent flow past a high-frequency in-line oscillating cylinder near free-surface," Int. J. Mod. Phys. C 27(12), 1650152 (2016).

${ }^{37}$ A. Zainali, N. Tofighi, M. S. Shadloo, and M. Yildiz, "Numerical investigation of Newtonian and non-Newtonian multiphase flows using ISPH method," Comput. Methods Appl. Mech. Eng. 254, 99-113 (2013).

${ }^{38}$ A. Rahmat, M. Barigou, and A. Alexiadis, "Numerical simulation of dissolution of solid particles in fluid flow using the SPH method," Int. J. Numer. Methods Heat Fluid Flow 30, 290-307 (2019).

${ }^{39} \mathrm{R}$. Rook, M. Yildiz, and S. Dost, "Modeling transient heat transfer using SPH and implicit time integration," Numer. Heat Transfer, Part B 51(1), 1-23 (2007).

${ }^{40} \mathrm{R}$. Saghatchi and J. Ghazanfarian, "A novel SPH method for the solution of dualphase-lag model with temperature-jump boundary condition in nanoscale," Appl. Math. Modell. 39(3), 1063-1073 (2015).

${ }^{41}$ J. Ghazanfarian, R. Saghatchi, and D. V. Patil, "Implementation of smoothedparticle hydrodynamics for non-linear Pennes' bioheat transfer equation," Appl. Math. Comput. 259, 21-31 (2015).

${ }^{42}$ A. Rahmat, M. Barigou, and A. Alexiadis, "Deformation and rupture of compound cells under shear: A discrete multiphysics study," Phys. Fluids 31(5), 051903 (2019).

${ }^{43}$ J. J. Monaghan and J. C. Lattanzio, "A refined particle method for astrophysical problems," Astron. Astrophys. 149(1), 135-143 (1985).

${ }^{44}$ J. J. Monaghan and A. Kocharyan, "SPH simulation of multiphase flow," Comput. Phys. Commun. 87(1-2), 225-235 (1995).

${ }^{45}$ J. P. Morris, "Simulating surface tension with smoothed particle hydrodynamics," Int. J. Numer. Methods Fluids 33(3), 333-353 (2000).

${ }^{46} \mathrm{~N}$. Tofighi and M. Yildiz, "Numerical simulation of single droplet dynamics in three-phase flows using ISPH," Comput. Math. Appl. 66(4), 525-536 (2013).

${ }^{47}$ M. Yildiz, R. A. Rook, and A. Suleman, "SPH with the multiple boundary tangent method," Int. J. Numer. Methods Eng. 77(10), 1416-1438 (2009).

${ }^{48}$ N. Tofighi, M. Ozbulut, A. Rahmat, J. J. Feng, and M. Yildiz, "An incompressible smoothed particle hydrodynamics method for the motion of rigid bodies in fluids," J. Comput. Phys. 297, 207-220 (2015).

${ }^{49}$ J. Q. Feng and T. C. Scott, "A computational analysis of electrohydrodynamics of a leaky dielectric drop in an electric field," J. Fluid Mech. 311, 289-326 (1996).

${ }^{50} \mathrm{~A}$. Rahmat and M. Yildiz, "A multiphase ISPH method for simulation of droplet coalescence and electro-coalescence,” Int. J. Multiphase Flow 105, 32-44 (2018).

${ }^{51}$ J. Zhang and D. Y. Kwok, "A 2D lattice Boltzmann study on electrohydrodynamic drop deformation with the leaky dielectric theory," J. Comput. Phys. 206(1), 150-161 (2005).

${ }^{52}$ A. Rahmat, N. Tofighi, and M. Yildiz, "The combined effect of electric forces and confinement ratio on the bubble rising," Int. J. Heat Fluid Flow 65, 352-362 (2017).

${ }^{53}$ T. Takao, Y. Yamamoto, T. Katayama, and M. Hozawa, "Effect of an electric field on the behavior of a drop moving in a quiescent liquid," J. Chem. Eng. Jpn. 27(5), 662-666 (1994).

${ }^{54}$ T. P. Hunt, D. Issadore, K. A. Brown, H. Lee, and R. M. Westervelt, Integrated Circuit/Microfluidic Chips for Dielectric Manipulation (Caister Academic Press, 2009). 